\title{
Indirect Angle Estimation in Switched Reluctance Motor Drives Using Fuzzy Logic Based Motor Model
}

\author{
Nesimi Ertugrul, Member, IEEE, and Adrian D. Cheok, Member, IEEE
}

\begin{abstract}
In this paper, a novel rotor position estimation scheme is described that was developed to overcome the drawbacks of the previous sensorless techniques, which were proposed for switched reluctance (SR) motor drives. It is based on fuzzy-logic, and does not require complex mathematical models or large look up tables. The scheme was implemented by using a digital signal processor. The real-time experimental results given in this paper exhibit that the position estimation method proposed can provide accurate and continual position data over a wide range of speeds (zero/low/high), and can also function accurately at different operating conditions (chopping/single pulse mode and steady state/transient operation).
\end{abstract}

Index Terms-Fuzzy logic, position sensorless operation, switched reluctance motor.

\section{INTRODUCTION}

$\mathbf{T}$ HE ACCURATE knowledge of the rotor position is required for good performance of the switched reluctance (SR) motor drive. The need for the rotor angle information in SR motors has been traditionally satisfied by the use of some form of rotor position sensor. However, in recent years, there have been extensive research activities to eliminate direct rotor position sensors, simply by indirectly determining the rotor position.

A comprehensive review of the existing indirect position detection methods in SR motors was discussed in the reference [1]. It has been shown in the reference that the indirect position determination methods can be classified into two major groups: inserting the low amplitude signals to the motor windings (major papers in this group include [2]-[4]), and monitoring the actual motor excitation waveforms (major papers in this group include [5]-[8], [28]). In [1], observations were also made about the disadvantages in the use of direct rotor position sensors.

The expected benefits of the indirect methods are: elimination of the electrical connections of sensors, reduced size, no maintenance, insusceptible to the environmental factors, and increased reliability. In addition, the expected features of the indirect methods over the other sensorless schemes should include: operating at zero speed and higher speed as in the conventional direct position sensors.

The method explained in this paper is a flux linkage based estimation method that uses a fuzzy motor model and estimation

Manuscript received April 8, 1999; revised February 21, 2000. Recommended by Associate Editor J. Ojo.

N. Ertugrul is with the Department of Electrical and Electronic Engineering, University of Adelaide, Adelaide 5005, Austrailia.

A. Cheok is with the Department of Electrical and Computer Engineering,

National University of Singapore, Singapore.

Publisher Item Identifier S 0885-8993(00)09797-0. scheme to determine the position of the rotor over a wide range of practical operating conditions. As well known, motor drives are usually electrically noisy environments, and practical measurement systems are normally subject to error and inaccuracy. Therefore, a major reason for the choice of using a fuzzy logic based estimation scheme was to satisfy the requirement that the algorithm is not affected significantly by deviations and error in the input data. The use of a fuzzy motor model in the estimation algorithm provided robustness and resistance to the effects of input noise, which is demonstrated in detail in reference [9], and therefore is not discussed here.

Furthermore, it should be noted that previously developed model based schemes [10]-[12] use simplified linear motor models and involve complex mathematical computations, or require large numerical look-up tables. This makes the previous schemes practically difficult to implement due to the fact that the SR motors normally operate under magnetic saturation and thus can only be accurately described by a nonlinear model. Moreover, complex mathematical computations are disadvantageous because of the demand for a fast real-time processor, which may not be suitable for all motor drives. Other advantages and applications of fuzzy logic to electric machine drives has been extensively detailed in [13].

The reference [14] reports the preliminary structure and hardware setup of the indirect position estimator. The paper presented some initial results to prove the concept, however only off-line results were presented that were calculated from measured voltages together with current waveforms derived from simulation.

This paper develops the scheme further to take into account the issues that are related to the practical motor drive operating in real-time and with measured voltages and currents. The hardware details of the DSP based system and the modifications introduced to provide a robust practical motor drive are also explained. In the following sections of the paper, the principal sections of the method are shown, implementation details are highlighted and some typical real-time experimental results are given to demonstrate the effectiveness of the method.

\section{Development of A Fuzzy Logic Based SR Motor MODEL}

To create a fuzzy model of the motor, a training scheme is used which trains a fuzzy logic model that is based on numerical information about the SR motor. The fuzzy rule base generated in this section is used by the fuzzy reasoning mechanism to estimate the rotor position from the input values of current and flux linkage in the rotor position estimation scheme. The main 
advantages of developing a fuzzy logic based model of the SR motor are as follows.

1) No complex mathematical model is required, and thus has the advantage of relatively simple mathematical calculations used for rule processing [15], and the memory requirement of the stored fuzzy model is much lower than that required by the equivalent look-up table [16]. In addition, the fuzzy motor models allow fast computation and hence provide cost effective solutions for computationally demanding algorithms in real-time systems.

2) Fuzzy models are universal approximators [17], and therefore, they can model a nonlinear continuous function of SR motor.

3) Neural networks can also be used to model the switched reluctance motor using a mathematical model free approach [18], but they often have a long learning time, and do not allow the examining of the internal structure of the model as with fuzzy logic linguistic rules [19]. Therefore, fuzzy models are favorable due to the fact that their behavior can be explained using linguistic rules, and thus, they can be easily adjusted by altering the rules [20]. Furthermore, it has been shown that the fastest possible universal computation scheme corresponds exactly to the operations in fuzzy logic methods using Max-Min computations [21], which allows faster real-time operation than is possible with an equivalent neural network model. This is an important consideration for practical SR drives.

\section{A. Obtaining the Motor Model}

To obtain a fuzzy rule based model of the test motor, the training system derives information from two main sources.

a) The static flux linkage curves of the motor, which provides important information about the electro-magnetic characteristics of the SR motor phases.

b) The dynamic real-time operating waveforms of the motor, which can include real-time operating effects, such as mutual coupling between phases, temperature variations, eddy currents, and skin effects.

Due to its suitability to practical applications (fast, simple, and accurate), the table-look-up scheme [15] was used for the training phase of the rotor position estimation system in order to derive a fuzzy logic based SR motor model in the form of a fuzzy rule base.

Furthermore, it should be emphasized here that the fuzzy model, which was implemented here, is not equivalent to a look up table with a linear interpolation, and has many advantages over look up tables: robustness to input noise [22], non linear model [17], much lower memory storage [16], and triggering multiple non linear rules for each numeric input (not just one rule as in a look up table).

Although the fuzzy rule generation techniques are well known, the rule generation for this specific application is briefly explained below to emphasize the practical issues.

The motor characteristics are defined as a two input (flux linkage and current) - one output (rotor angle) function. The training task involves creating a fuzzy model of this function from the training data. The training data is defined as a two-input one-output input-output pair (where the word "pair" in this term refers to the fact that there is a set of input values paired with a set of output values, and not a pair of two values). Each point of measured data presented to the training system is given as

$$
\left(\psi^{(n)}, i^{(n)} ; \theta^{(n)}\right)
$$

where

$n \quad n$th data pair;

$\psi \quad$ flux linkage;

$i \quad$ current;

$\theta$ position.

The training phase to obtain a fuzzy logic based motor model consists of the following steps.

Step A: Dividing the Input and Output Domains into Fuzzy Regions: To determine the fuzzy regions, the variable spaces of $\psi(0$ to $1 \mathrm{~Wb}), i(0$ to $20 \mathrm{~A})$, and $\theta(0$ to 30 degrees $)$ were divided into $N_{1}, N_{2}$, and $N_{3}$ regions respectively, and number of regions were chosen to be $N_{1}=39, N_{2}=37, N_{3}=31$.

Note that the number of sets and all fuzzy sets were chosen to have the same shape, and thus all the membership functions were chosen to be isosceles triangular shapes, which were defined after the real-time testing of the algorithm in the realsystem. This choice of sets was found to provide sufficient accuracy in this work. Although more regions would provide greater accuracy in such systems, this also leads to more memory requirement due to the greater number of fuzzy sets and rules.

Each region was then assigned to a fuzzy membership function. The maximum point of each triangle was chosen to lie at the center of the fuzzy region and is given a membership value of 1 . The other two vertices were chosen to lie at the centers of the two neighboring fuzzy regions and at these two points the membership values were made zero. Each fuzzy set is denoted by a fuzzy linguistic term ranging from set SM19 to BIG19 (for $\Psi^{\prime}$ ), from set SM18 to BIG18 (for $I^{\prime}$ ), and from set SM15 to BIG15 (for $\Theta^{\prime}$ ) as shown in Fig. 1. In the figure, $\mu_{\Psi^{\prime}}(\psi)$ is the membership value in fuzzy set $\Psi^{\prime}$ of input flux value $\psi, \mu_{I^{\prime}}(i)$ is the membership value in fuzzy set $I^{\prime}$ of input current value $i$, and $\Psi_{\Theta^{\prime}}(\theta)$ is the membership value in fuzzy set $\Theta^{\prime}$ of input angle value $\theta$.

Step B: Generating Fuzzy Rules from Input Data of Flux, Current, and Angle: During the training phase, each input-output data pair, which consists of a crisp numerical value of measured flux linkage, current, and angle, is used to generate the fuzzy rules which model the system. To determine a fuzzy rule from each input-output data pair, the first step is to find the degree of each data value (flux, current, angle) in every membership region of its corresponding fuzzy domain. The variable is then assigned to the region with the maximum degree.

It should be mentioned here that each training data set produces a corresponding fuzzy rule, which is stored in the fuzzy rule base. However, it can be seen that with a large amount of measured training data there will normally be rules produced by different training data which are identical, and therefore the number of stored rules does not necessarily correspond to the number of training data sets. In addition there may be rules 


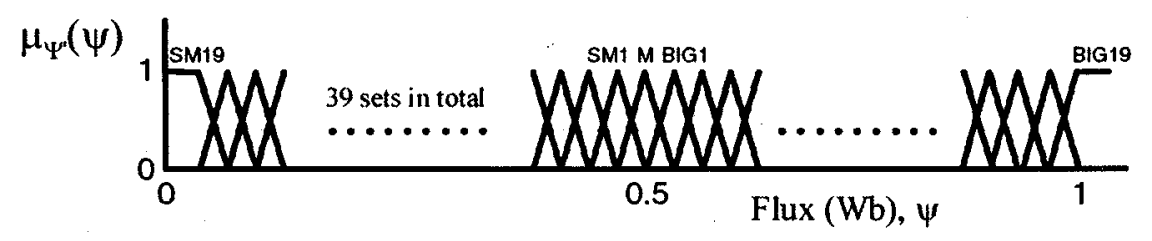

(a)

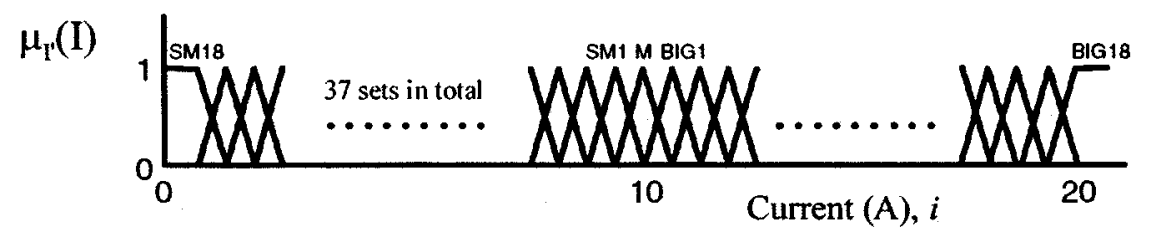

(b)

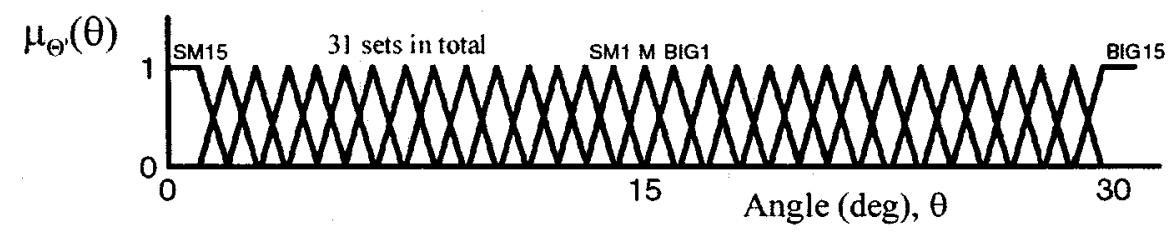

(c)

Fig. 1. Fuzzy domain regions for each variable: (a) flux Linkage, (b) current, and (c) rotor position.

generated by different data sets that are contradictory, and the method for dealing with this case is explained below.

Step C: Assigning Rule Degrees: When each new rule is generated from the input-output data pairs, a rule degree or truth is assigned to that rule, where this rule degree is defined as the degree of confidence that the rule does in fact correlate to the function relating flux linkages and current to angle. In the developed method a degree is assigned which is the product of the membership function degree of each variable in it's respective region. For example

$$
\begin{aligned}
& \text { "Rule): If } i \text { is BIG2 and } \Psi \text { is BIG5 Then } \theta \text { is SM6" } \\
& \text { will have a degree } \\
& \text { "Degree (Rule) }=\mu_{\mathrm{BIG} 2}(i) . \mu_{\mathrm{BIG} 5}(\Psi) \cdot \mu_{\mathrm{SM}}(\theta) "
\end{aligned}
$$

where

$\mu_{\mathrm{BIG} 2}(i) \quad$ membership degree of current in region BIG2;

$\mu_{\mathrm{BIG} 5}(\psi)$ membership degree of flux in region BIG5;

$\mu_{\text {SM6 }}(\theta)$ membership degree of angle in the region SM6.

The purpose of the above assignment is to choose between data sets that produce the same antecedents but different consequents. This would arise because when there is a large amount of measured data, some data pairs will produce rules that have the same antecedent but a different consequent (due to errors or noise in the measured data). This would mean that there are conflicting rules in the system, which are resolved by choosing the conflicting rule that has the highest degree. This rule is the one that is placed in the fuzzy rule base.

For example, let us consider two input-output data pairs $n$ and $m$, which produced the rules

Rule $(n)$ : If $i$ is BIG2 and $\Psi$ is BIG4 Then $\theta$ is SM7

Rule $(m)$ : If $i$ is BIG2 and $\Psi$ is BIG4 Then $\theta$ is SM9.
Thus for this example, there would be two rules with the same precedent but different consequent. If the membership functions for each of the variables was as follows:

i) Data Set $n: \mu_{\mathrm{BIG} 2}\left(i^{(n)}\right)=0.41, \mu_{\mathrm{BIG} 4}\left(\Psi^{(n)}\right)=0.73$

$$
\mu_{\mathrm{SM}}\left(\theta^{(n)}\right)=0.79
$$

ii) Data Set $m: \mu_{\mathrm{BIG} 2}\left(i^{(m)}\right)=0.92, \mu_{\mathrm{BIG} 4}\left(\Psi^{(m)}\right)=0.76$

$$
\mu_{\mathrm{SM} 9}\left(\theta^{(m)}\right)=0.80 \text {. }
$$

Then the rule $n$ will have degree $0.41 \times 0.73 \times 0.79=0.236$, whilst rule $m$ will have degree $0.92 \times 0.76 \times 0.80=0.559$. Therefore, only the rule with the highest degree $(m)$ will be placed into the fuzzy rule base.

Step D: Create the Fuzzy Rule Base: As it can be seen from Step C, every training data set produces a corresponding fuzzy rule that is stored in the fuzzy rule base (except if an identical rule exists on the rule base already, or the generated rule is eliminated due to a lower degree of truth than an existing rule with the same antecedent but different consequent). Therefore, as each input-output data pair is processed, and the rules are generated, a fuzzy rule or knowledge base is in the form of a two dimensional table, which can be looked up by the fuzzy reasoning mechanism. The current and flux linkage fuzzy sets, which are the antecedents, are the axes of a two dimensional look-up table, and the stored table values are the rotor position output sets.

\section{B. Implementation of Training Scheme}

A flow-chart showing the logical flow of the training procedure software routine is given in Fig. 2 [23], which is the same for both phases of training (using the static training data and the dynamic real time training data). The training algorithm learns the motor model from the two sets of measured motor data: the static magnetization curves and the real time dynamic operation data. After training the system with all the points on the static flux linkage curves, the rule table is generated. 


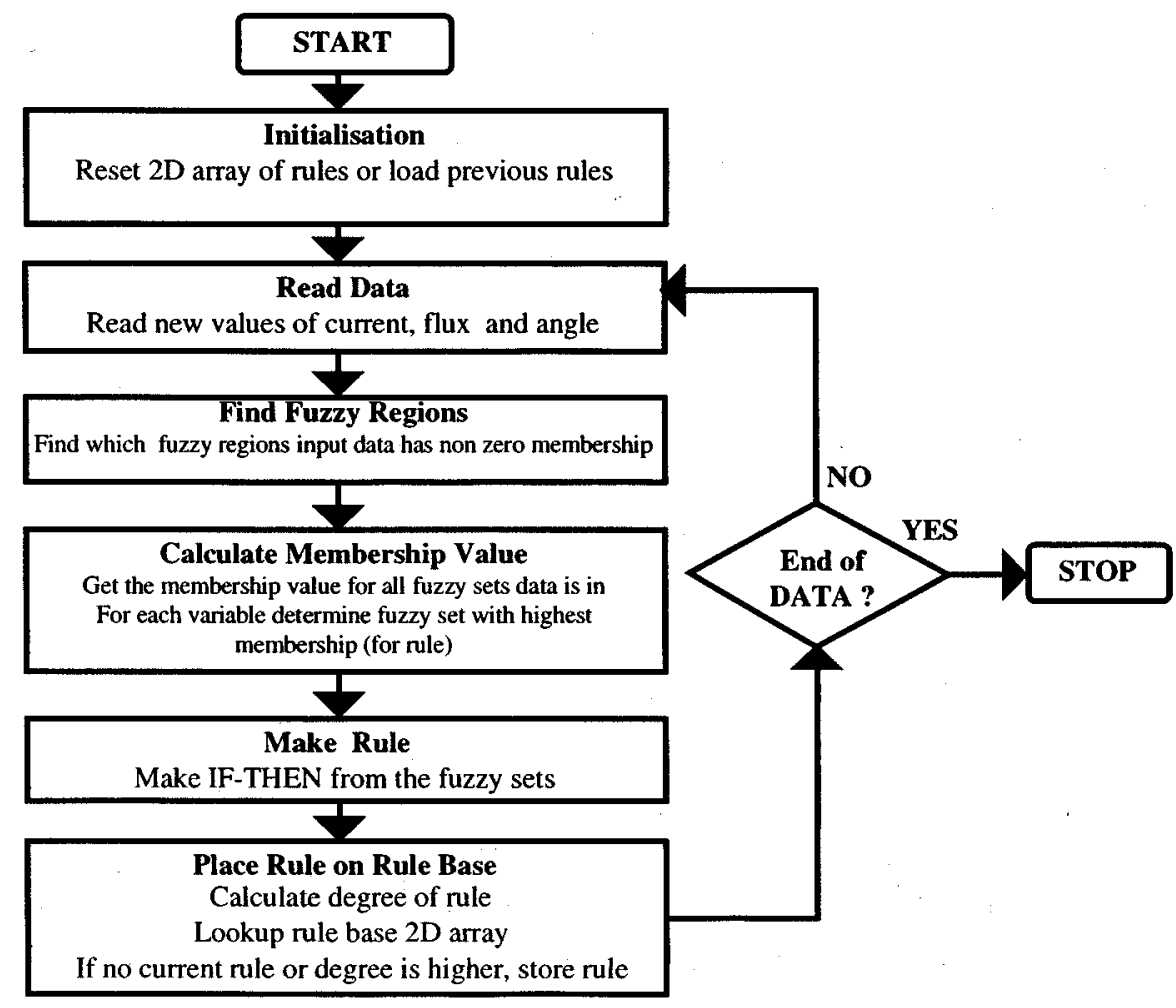

Fig. 2. Flow chart for the training algorithm.

It was found out that the static flux linkage curves characterize the motor to a good degree. However, the real time operating effects such as mutual inductances between phases, eddy currents, temperature effects and skin effects may be significant. It was found that there are some empty rule areas which cannot be explained by the rule table obtained as they lie in the region of flux and current in which the curves lie, and therefore, the dynamic testing requirement for the rule base determination is found to be necessary.

To determine the real time operational points of the SR motor it is important that data is measured during the real-time training phase include a wide range of operating conditions: transient and steady state speeds, step changes in load, and chopping and single pulse mode.

After the real-time running tests, the modifications made to the rule base from the static data training phase are highlighted (boxes with black background) in Fig. 3. The empty rule areas are shown by "XX" in the figure. For example, there is no rule for the inputs "Current is SMALL16 and Flux is BIG10."

It should be reported here that the amount of data that is required to be measured in the dynamic tests and used for training data cannot be exactly specified. However, it can be said that the accuracy of the model, up to a point, will increase with an increase in the data processed during the training phase. This is important because if data from a wide range of real time conditions is used for training, then the developed motor model will be able to predict the rotor position more accurately from the real time measurements of current and flux. Furthermore, it should be emphasize here that all the measurements in the motor are done for a single phase of the four-phase SR motor, and as a result, a single table is produced. Therefore, it can be said that the model developed here evolve an "average model" by using the mechanism of maximum degree of truth.

In addition to this, note that fuzzy sets are defined over a range of values, with the membership function of the fuzzy set varying for different values in the range. This means that an input data point with error or noise can still be placed, with lower membership function, in the same set as a point with no error or noise (depending on the amplitude of the error). In other words, by the use of fuzzy sets, input data that is corrupted by noise, can be accepted into the same set as clean data but with a lesser degree of truth [24]. The length of range of the membership function will determine the range of values with noise, which will be accepted as a part of the fuzzy set. Therefore by the fuzzification of the input data, small deviations in the input data do not have significant effect on the output position estimation.

\section{IMPLEMENTATION OF THE COMPLETE ROTOR ANGLE DETECTION SCHEME}

\section{A. Position Estimation}

A block diagram of the complete position estimation algorithm is shown in Fig. 4. The position estimator essentially operates as follows: While the motor is running, the phase currents and voltages in each of the four phases are measured and the flux linkages are estimated by using trapezoidal integration (see the block A in Fig. 4) as given by

$$
\begin{aligned}
\psi(n+1)= & \psi(n)+\Delta T \\
& \cdot[v(n)-R i(n)+v(n-1)-R i(n-1)] / 2 \\
\psi(0)= & 0
\end{aligned}
$$




\section{Flux Linkage}

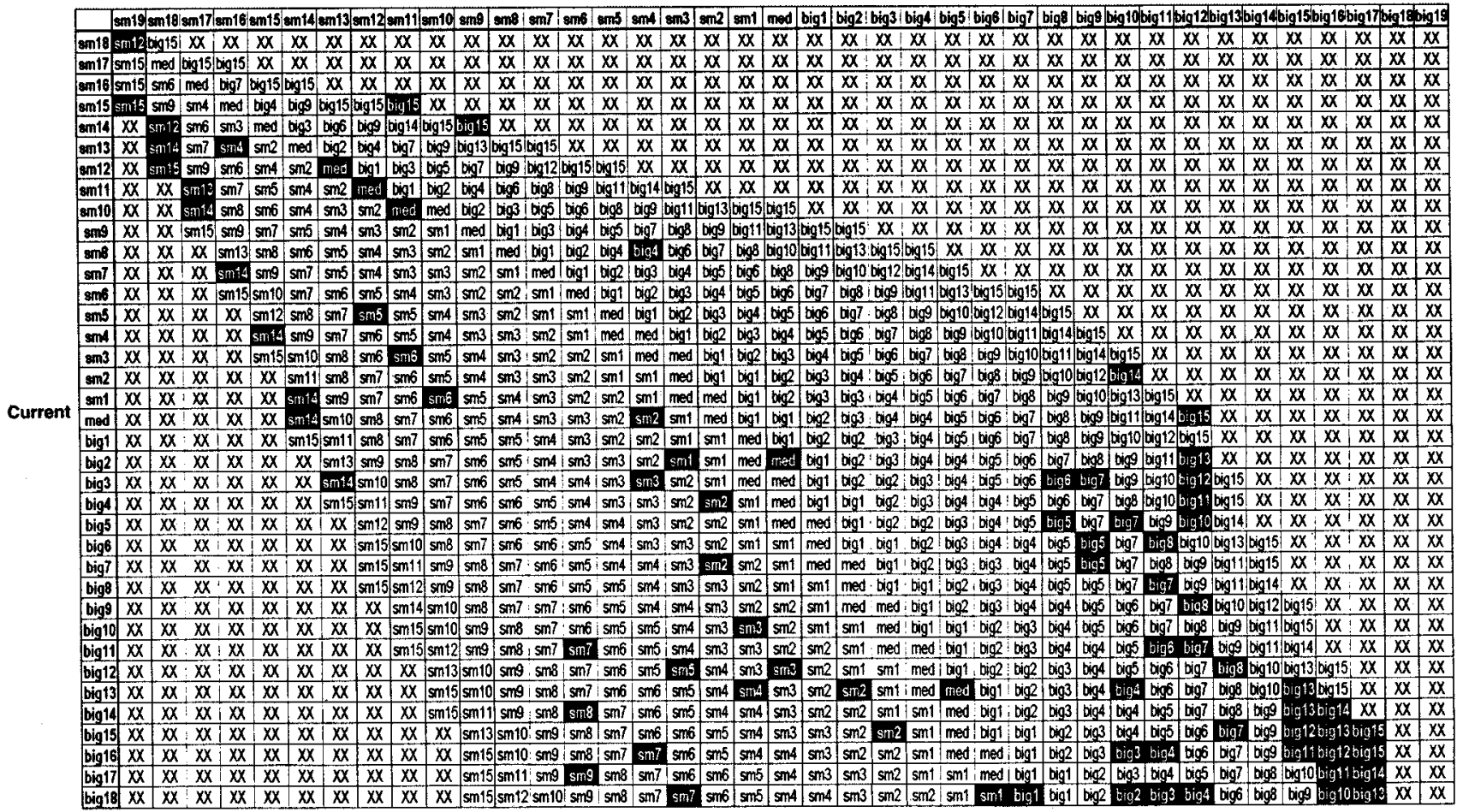

Fig. 3. Fuzzy rule bases generated after the real-time test results, S: small, C: center, B: big.

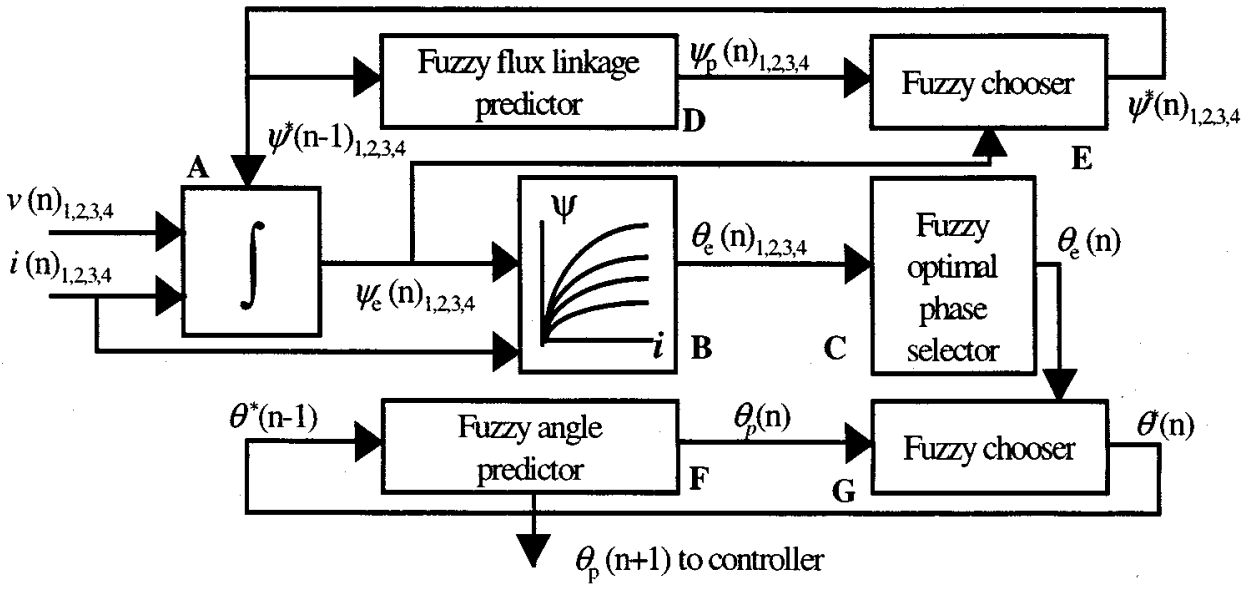

Fig. 4. Block diagram of the fuzzy logic based position estimation scheme.

where

$n$ sample number;

$\Delta T$ sampling period;

$v \quad$ winding voltage;

$i \quad$ line current;

$R \quad$ winding resistor.

Note that when no current flows in a SR motor phase, the initial flux linkage value at $t=0$ is zero. Therefore, at the end of each electrical cycle for each phase the flux linkage can be reset to zero in the integrator. Unlike the other three-phase ac motor drives, this feature of SR drive flux linkage estimation provides a means of reducing the effect of flux linkage offset error [9]. This is because the accumulation of the large errors in each phase, such as the effects of currents and voltage dc offset, measurement errors and resistance variation, will not normally continue for more than one electrical cycle.

It should also be noted here that the maximum integration error is also proportional to the highest value of the second derivative of the integrated function [23]. This means that when trapezoidal integration is applied to the flux linkage estimation, the maximum error is dependent on the second derivative of the integrated current and voltage waveforms.

It should be emphasized that, the current and voltage waveform are simple functions at high motor speeds (in the single pulse mode). However due to the commutation seen in the chopping mode, the current and voltage waveforms are more complex and nonlinear. Therefore, for the same conditions, it can be expected that the error in the integration of flux linkage in the 
chopping mode may be higher than in the single pulse mode (in which the integration intervals is wider and the second derivative is lower).

In the above figure, $\psi$ is the flux linkage, 1,2,3 and 4 represent the phase numbers, and $\theta$ is the rotor angle. $n, n-1$, and $n+$ 1 indicate "present," "previous" and "next step" values of the parameters respectively. The superscript, $*$ and the subscripts, $\mathbf{p}$ and $\mathbf{e}$ in the terms indicate the weighted values, the predicted values and the estimated values respectively.

In the second stage, the block $\mathrm{B}$, the crisp numeric flux linkage and current values from each phase are then input to the fuzzy logic rule base (where the fuzzy motor model is located). These inputs will trigger the If-Then rules in the rule base, which have previously been created from the training phase. Since the crisp values of current and flux linkage will normally be members of two fuzzy sets, four rules will normally be triggered per phase measurement. Therefore, composition of multiple fuzzy rules is required, using the Aggregation of Rules procedure. The aggregate of the fuzzy rules triggered by the inputs of each phase will produce an output fuzzy set in the rotor position fuzzy domain. A single crisp numeric value of estimated rotor position is obtained by using defuzzification. In this paper, the Max-Product and center average defuzzification methods are chosen due to the simple calculations of these methods [15].

It should be noted here that, in practice, drive environments are electromagnetically noisy, due to the proximity of power electronic devices, which have high amplitude voltage and current switching transient waveforms, with low power computation circuits. In addition, leakage inductances and coupling capacitances, which are always finite in such systems, can lead to noise voltages being induced in measurement circuits. Therefore, the reliability and robustness of the algorithm was highly important if it is designed to operate in the practical drive. To achieve this additional performance enhancement features have been added so that the sensorless angle estimation algorithm copes better with measurement errors and inaccuracies found in real motor drives.

\section{B. Performance Enhancement Features}

Fuzzy Optimal Phase Selector: The knowledge based optimal phase selector is added (the block $\mathrm{C}$ in Fig. 4) to pick the most desirable phase for estimating angle in order to maximize accuracy. This sub-system is used because in the SR motor there may be more than one phase that conducts excitation current at any instant of time. For example, if three phases were conducting current at a given instant in time, this would normally consist of two phases being turned on, with one previously excited phase having a decaying current component. Therefore, any of these current carrying phases may be used for rotor position estimation. Theoretically, the same position should be output by each of the excited phase rotor position estimations. However in practice, each phase may produce a slightly different estimated angle result.

It is important to note that each phase has rotor angle regions of optimal sensing. In some rotor angle regions the rotor position estimation will be more affected by errors than at other rotor

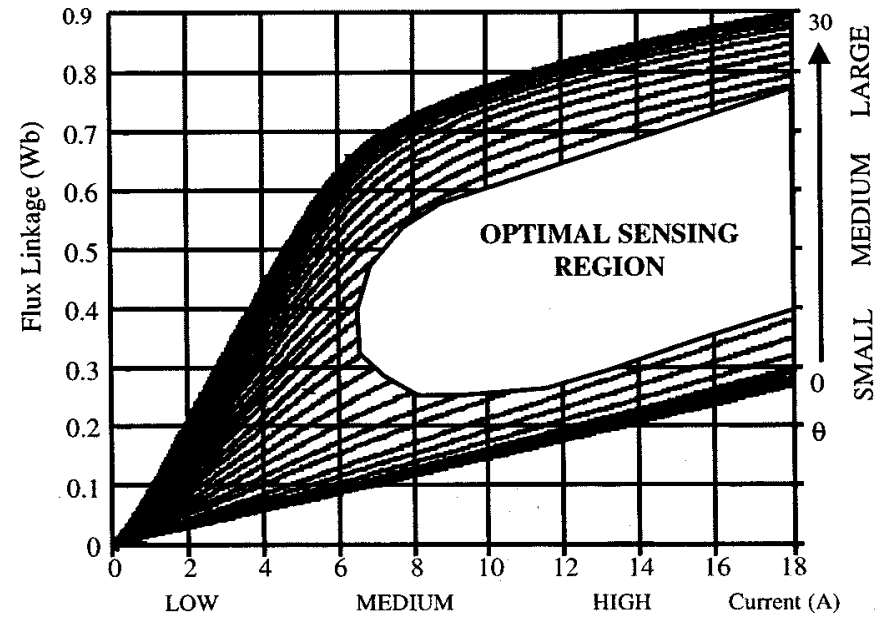

Fig. 5. Approximate optimal sensing region of the magnetization curves.

angle regions. The reason that each phase will be operating in a different phase region at any point in time is that at every physical rotor position, the rotor to stator phase angle will lie in a different region in each motor phase. Therefore, for implementation in a practical drive, the estimated rotor position from the phase in the rotor angle that lies in the optimal region should be given the most weighting. The optimal sensing region in Fig. 5 can be found from an analysis of the flux linkage curves [25].

Note also that the region shown in Fig. 5 is only approximate, as it is difficult to exactly define in an exact manner. It can generally be said from the magnetization curves, that when the angle is near the unaligned position (ie. for small relative angles) and the current is low, that the curves are very tightly bunched up. Therefore under this condition, small errors in the flux linkage estimation or current measurement will result in large errors in the position estimate. Additionally, when the angle is close to alignment (i.e., large relative angles) the curves are also tightly bunched up, and therefore a small error in current measurement can produce large errors in rotor angle. Therefore, the optimal sensing positions, where the best resolution is offered, is for medium angles between alignment and unalignment.

However, the optimal sensing region in fact does not stop or start abruptly but has a transition region, or in other words it contains smooth edges. In addition, the terms small, low, medium, and large used above to describe the regions, are linguistic terms. Furthermore it can be seen that the above descriptions in the previous paragraph describing the optimal sensing regions were in fact heuristic knowledge based rules.

Thus due to the imprecise regions, the ability to describe them using linguistic terms, and the availability of heuristic rules, a fuzzy logic rule base can be employed. Hence, to decide whether a motor phase measurement is in the optimal sensing region, a fuzzy rule based optimal phase selector (Block C) is placed after the fuzzy logic based motor model (Block B) seen in Fig. 4. The decision block encapsulates the general heuristic rules that were mentioned above, which describe the optimal sensing regions of the motor phases.

To perform the weighting of each of the position estimations from each phase, based on the heuristic rules described above, the optimal phase selector, uses a two input-single output fuzzy 

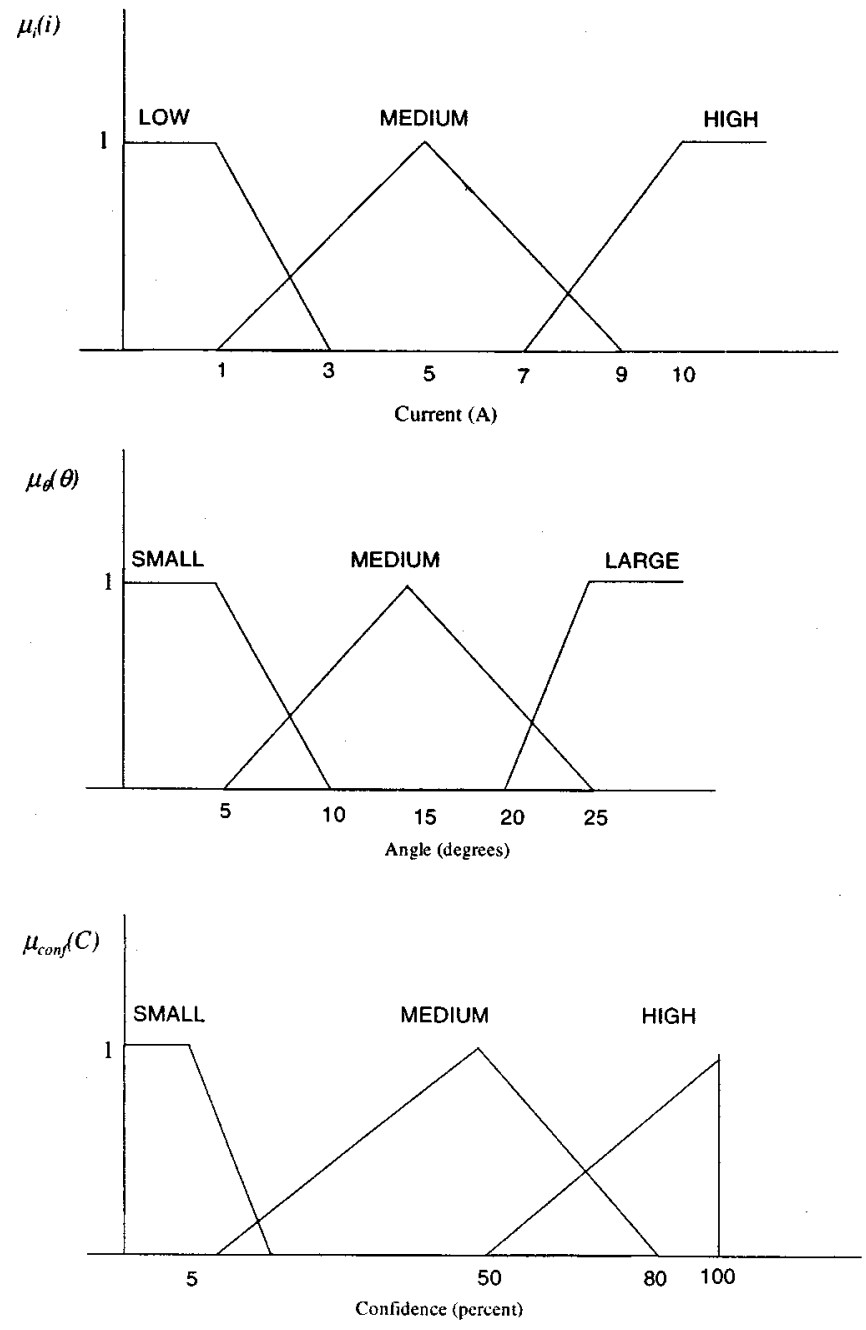

Fig. 6. Optimal phase selector domains. $\left[\mu_{i}(i)=\right.$ fuzzy membership functions of current. $\mu_{\theta}(\theta)=$ fuzzy membership functions of rotor angle. $\mu_{\text {con } f}(C)=$ fuzzy membership functions of confidence.]

system. The input fuzzy domain is current and angle and the output is confidence. It gives a weighting or confidence value ranging from zero to $100 \%$ of each phase's rotor position estimate. The membership functions of the input and output fuzzy domains used in the optimal phase selector are shown below in Fig. 6.

A fuzzy rule base defines the linguistic rules that are used by the optimal phase selector. This is shown in the two-dimensional array of rules relating the inputs of current and angle to the output confidence value in Table I. It can be seen from the rule table that an example rule in this system is

If current is $S M A L L(\mathrm{~S})$ and angle is $S M A L L(\mathrm{~S})$

Then confidence is $S M A L L(\mathrm{~S})$.

As it was discussed above, these rules are based on heuristic knowledge about the optimal sensing regions in the flux linkage curves. The ability of fuzzy logic to model this heuristic knowledge allows a simple and easy to understand linguistic based system to be easily developed. This is achieved without the requirement of analytically defining the optimal regions of position sensing.
TABLE I

FuZzy Rule Base For Optimal Decision Block ( $\mathrm{S}=$ SMALl, $\mathrm{M}=$ MEDIUM, L = LARGE, $\mathrm{H}=\mathrm{HIGH}$ )

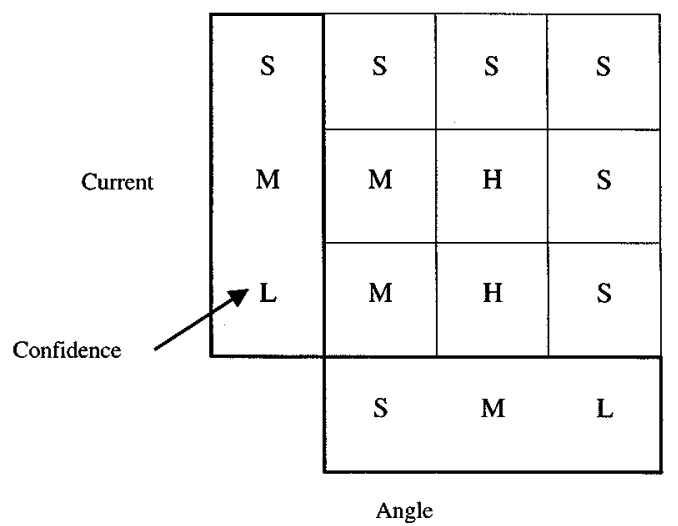

Another advantage of using the fuzzy system is that the fuzzy linguistic rules of the optimal phase selector can remain unchanged even when a different motor is used. This is because only the definitions of the fuzzy membership functions in the input domains of current and angle are required to be changed if there is a change in the motor with different optimal phase sensing regions. For example, the membership function of $L O W$ current seen in Fig. 6 could be modified to lie over a different range of the current domain. However, the fuzzy rule base does not need to be changed, and this allows the modification of the Optimal Phase Selector Block for another motor to be easily achieved.

As it was mentioned above, each of the four motor phases produces an estimated value of position, and each of them will be given a confidence weighting by the fuzzy phase selector, based on the estimated position and the phase current. This final weighted value is based on the weighting or confidence, $\mathrm{C}$, of each of the rotor position estimates corresponding to which region the estimated rotor position lies in each phase. In essence, the optimal phase selector block in Fig. 4 decides the optimum phase for angle measurements, and gives this phase the greatest weighting if more than one phase is used in producing a rotor position estimate. Hence $\theta_{e}(n)_{1,2,3,4}$ outputs one final weighted value of $\theta_{e}(n)$.

To determine the final angle value $\theta_{e}(n)$, each estimated phase angle is multiplied by its respective confidence value found from the Optimal Phase Selector, and the total is divided by the addition of all the confidence factors. For example, if there are two phases that produce an angle estimate, then the algorithm computes the position by

where

$$
\theta_{e}=\frac{\theta_{1} \cdot C_{1}+\theta_{2} \cdot C_{2}}{C_{1}+C_{2}}
$$

$\theta_{e}$

$\theta_{1}$ and $\theta_{2}$ phase angle estimates of phase 1 and phase 2 , respectively;

$C_{1}$ and $C_{2}$ confidence values of phase 1 and phase 2 , respectively.

It should also be noted here that (4) is given only for two conducting phases. If three, or in some cases, four phases have some current (for example, trail currents, at high-speed operations), the equation should be modified to take these operating conditions into account. 


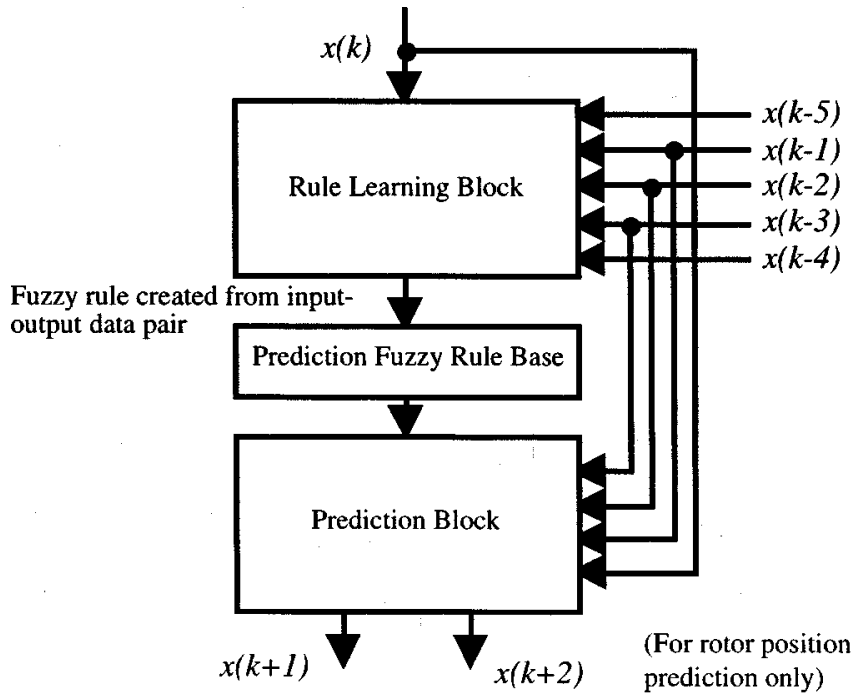

Fig. 7. Flow-chart of the prediction algorithms in the blocks D and F.

Fuzzy Flux Linkage and Angle Predictors with Fuzzy Choosers: The flux linkage and rotor angle predictors (the blocks D and F in Fig. 4) are included in the algorithm. The purpose of the predictors is to forecast future values of flux linkage and rotor position during the operation of the sensorless algorithm. The predictors are implemented using a fuzzy logic rule based system, with the rules of the fuzzy prediction system being adapted during run time.

The predictors are used to minimize errors by using a combination of the estimated and predicted rotor position and flux. To achieve this, a comparison between estimated and predicted rotor position and flux values are made during each iteration. Then some combination of these is chosen in order to lessen the effect of errors.

The problem of predicting the flux linkage and angle in future steps of time is a problem of time series prediction. It should be noted that in this fuzzy predictor, both learning and prediction occurs simultaneously, unlike the previously described training method. A flow-chart of the prediction algorithm is given in Fig. 7, where the values of $x(k)$ represent either flux linkage or rotor position.

When a new value of flux linkage or rotor position is estimated by the integrator or fuzzy model respectively, it is first passed into the rule learning block in Fig. 7, together with previous iteration values. For the flux linkage prediction, the rule learning block creates a new rule and modifies the rule table, from the new input-output data pair consisting of the present value $x(k)$ and the previous four values $x(k-1), x(k-2), x(k-3)$, and $x(k-4)$. For the fuzzy rules of the rotor predictor, however, two output predicted values, $x(k)$ and $x(k-1)$ are created from four previous inputs $x(k-2), x(k-3), x(k-4)$, and $x(k-5)$. For the flux prediction and the rotor position prediction, the input-output data pairs can be written, respectively, as

$$
\begin{gathered}
{[x(k-4), x(k-3), x(k-2), x(k-1)], \rightarrow[x(k)]} \\
{[x(k-5), x(k-4), x(k-3), x(k-2)], \rightarrow[x(k-1), x(k)] .}
\end{gathered}
$$

This rule can then modify the fuzzy rule base so that the fuzzy rules which predict the next values from the previous four values is continuously adapted with each new measurement.

After the Rule Learning Block in Fig. 7, the prediction routine is executed, which estimates the next iteration value $x(k+1)$ (and $x(k+2)$ for the angle prediction), from the values of $x(k), x(k-1), x(k-2)$, and $x(k-3)$.

Furthermore, as shown in Fig. 4, the predicted values of rotor position $\theta_{p}(n)$, and flux linkage $\psi_{p}(n)$ are used in conjunction with the estimated values of flux linkage $\psi_{e}(n)$ and rotor position $\theta_{e}(n)$. In the ideal case, the predicted and estimated values should be exactly the same. However, due to errors the values are not equal. In this case either the predicted values or the estimated values may be used, and a decision must be made as to which value should be chosen. In this system a knowledge based, heuristic decision maker (Fuzzy Chooser) was implemented, which places a weighting on both the predicted and estimated values (the blocks E and G in Fig. 4). The decision blocks of the flux linkage and angle produce a final weighted value $\psi^{*}(n)$ and $\theta^{*}(n)$ respectively.

It can be intuitively said that the confidence in predicted values will be high under steady speeds and conditions. Under transient speeds and conditions, however, confidence will be low. In addition, it can be said that the confidence in the predicted values will be higher for low acceleration values than for high acceleration values.

From the above discussion it may seem that some conventional mathematical function relating confidence in the predicted values to the actual motor acceleration can easily be defined. However some practical considerations make the use of a fuzzy system advantageous.

Firstly it can be seen from the above discussion, that high, low, and steady are linguistic terms that contain a certain amount of fuzziness. With conventional mathematical logic functions it is difficult to adequately represent heuristic knowledge directly. However, fuzzy systems can deal with situations where sharp distinctions between the boundaries of application of rules do not occur.

Furthermore, a major advantage of using a fuzzy system is that it can cope with inherent uncertainty in the input signals. In this system the input variable is acceleration, which cannot be directly measured by a mechanical sensor in this application, because the system is sensorless. Another method is to calculate acceleration from speed values. Successful techniques have been recently developed to estimate acceleration from measured speed, such as by using predictive polynomial differentiators or by model based state observation [27]. However in this case no direct measurement of position or speed is possible. If the position estimates are used instead, the errors in the estimates may be too high for calculation of acceleration.

Therefore a fuzzy system was developed to relate prediction confidence to acceleration, which only requires the acceleration feedback to be accurate enough to determine which predefined fuzzy domain the acceleration belongs to. Thus only imprecise knowledge is required about the motors acceleration.

Hence, instead of acceleration, an acceleration factor is used which gives an approximation of the actual acceleration. As discussed above, this approximation can be used in this application 


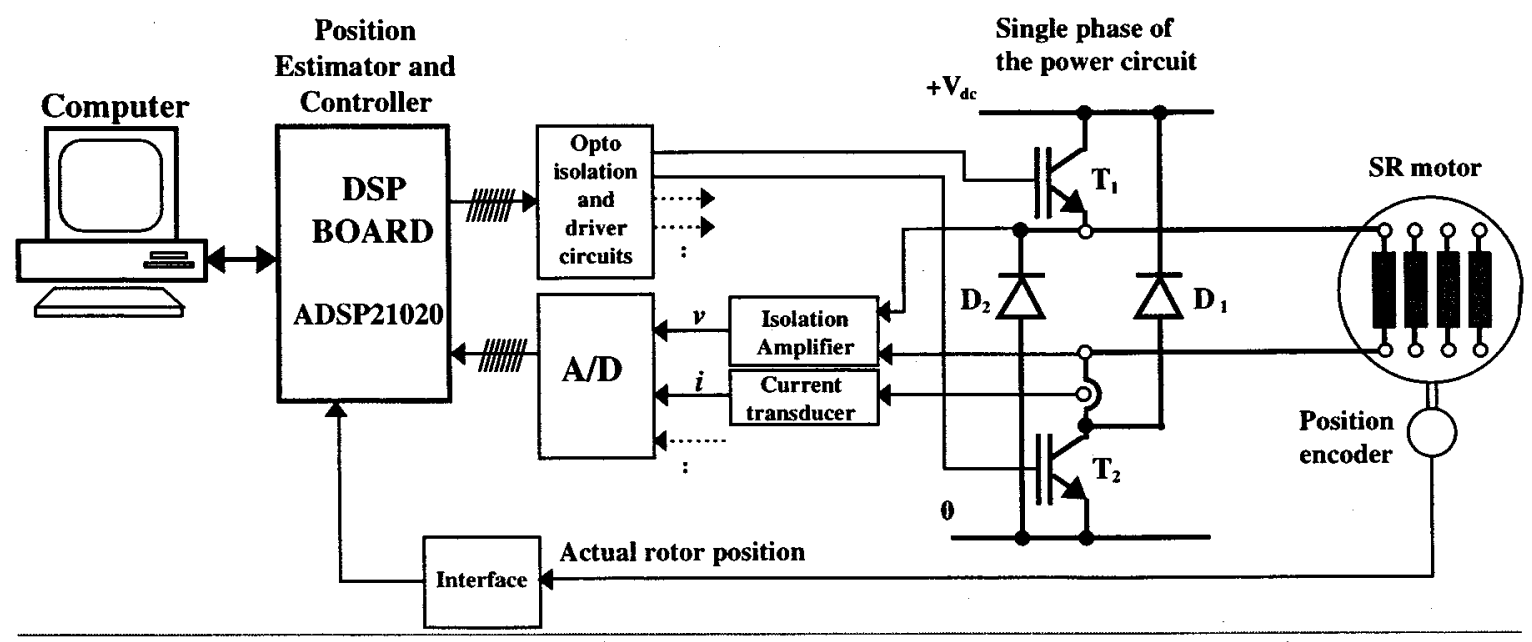

Fig. 8. SR motor drive hardware system developed.

because only the relative acceleration is important (e.g., high or low), and not the actual numeric value. Therefore an acceleration factor $A_{n}$ is defined as

$$
A_{n}=\frac{\theta_{n}-\theta_{n-k}}{k \Delta T}-\frac{\theta_{n-l}-\theta_{n-k-l}}{k \Delta T} .
$$

Here, $\theta_{n}$ is the rotor angle at step $n$ and $\Delta T$ is the time between each iteration, and $k$ and $l$ are constants (each chosen to be 5 in this application).

The above equation estimates the average speed over the last six iterations (i.e., over iteration $n$ to $n-5$ where $n$ is the last estimated position value), and compares it to a previous estimated average speed measured over $n-5$ to $n-10$ ). Other values of $k$ and $l$ may be chosen depending on the desired tradeoff between a longer sampling time of measuring the acceleration factor (which leads to less noisy values) and the delay in determining the value from the first measurement $\theta_{n-k-l}$.

The difference of these two values provides an estimate of the relative motor speed transient magnitude. However it was found out in the practical system that, to lessens the effect of angle estimate noise on the calculation, the acceleration factor could be further modified to use the average of the previous 3 estimated acceleration factors, which is given by

$$
\left(A_{n}+A_{n-1}+A_{n-2}\right) / 3 \text {. }
$$

The weighted rotor angle from the estimated and predicted value is used as an input to the rotor position predictor in Fig. 4, in order that further predictions are corrected. In addition, the flux linkage value output from the decision block is used as the next integration constant for the flux linkage integrator. In this manner, the corrected values are used to not only correct the present values of flux linkage and position, but also to correct future values of flux linkage and position. The effectiveness of the flux linkage and angle prediction with fuzzy choosers in reducing the effect of impulsive type noise commonly found in motor drives is detailed below.

\section{IsSUes RELATEd to START-Up, INITIALIZATION AND NEXT STEP ANGLE}

In the method developed, there are some practical application points, which apply in all operations in the drive. This includes issues such as the starting procedure, the initialization of the fuzzy predictors in each test, and the use of predicted angle when the fuzzy logic based predictors do not output a predicted angle due to lack of rules in the learning period. These issues are explained below.

\section{A. Start-Up Procedure}

During the start up of the sensorless motor drive, there are two problems. Firstly, the position is not known, and therefore, the controller does not have knowledge of the required initial phase control strategy. Secondly, if only one phase is used initially, there will be two solutions to the estimate of rotor position for each flux linkage and current data pair.

In the initial starting instant, two phases of the motor are excited using a short pulse of current, to produce two sets of flux linkage and current pairs. This will produce four possible values of absolute position (two from each motor phase). Only one angle estimate of one phase will agree with one angle estimate of the other phase. This is the actual absolute rotor position. After this step is performed, the absolute value of rotor position has been found, and there will be no ambiguity in further measurements. This is because knowledge of the last rotor position, and the direction of rotation, allows the controller to decide which of the two possible angles found from a flux linkage and current pair is in fact the correct position.

\section{B. Initialization of Fuzzy Predictors}

The fuzzy predictors of flux linkage and rotor position are initialized at the beginning of each of the tests. Therefore, in each of the graphs that are plotted, at time $t=0$ there are zero rules in the fuzzy prediction rule base and learning begins with the first iteration. This is performed so that every test shown in the results section has a fair comparison, regardless of whether the motor starts at $t=0$ (as in the start up tests) or not (as in the steady-state speed tests). It should be remembered that, this 
arbitrarily places a learning period at the initial stages of each test, regardless of the motor operating conditions.

In addition, when the predictors cannot make a predicted value, due to a lack of developed rules in the learning system, the output is set to zero. This signifies that no prediction can be made at that particular iteration (in addition there is a software flag output implemented in the software to differentiate between no output, and an actual predicted value of zero degrees). If no prediction can be made for the current angle and flux linkage, then it is not of high consequence. However, if no prediction can be made for the next step angle, then this creates a problem for the controller, which uses the next step for the continuous control. Therefore, a backup system is used for the prediction of the next angle value.

\section{Use of Next Step Predicted Angle in Fuzzy Predictor}

When the predictors can not make a prediction, the output is set to zero. However, although the fuzzy predictor of flux linkage and rotor position is only required for error minimization, the next step angle is used by the controller. Therefore, in the case when the output of the next step angle is zero, the prediction reverts to a simpler prediction scheme which is independent and running in parallel to the predicted value.

The independent next step angle prediction acts essentially as a backup, and normally is not used by the controller, except when the predictor cannot make any prediction. This independent next step angle predictor performs a linear extrapolation of the previous two iteration's angle rotor position values. The value of the predicted angle can be found from the simple relation

$$
\theta_{n+1}=\theta_{n-1}+2 \frac{\left(\theta_{n}-\theta_{n-1}\right)}{\Delta T}
$$

where $\theta_{n+1}, \theta_{n}, \theta_{n-1}$ are the predicted angles of the next iteration step, the present iteration step, and the previous iteration step, respectively, and $\Delta T$ is the iteration period.

The predicted angle using this method is normalized to one electrical cycle (60 degrees). The new angles are used for prediction in the next iteration.

\section{Hardware Details and Real-Time Test Results}

To test the method described in this paper, a switched reluctance motor drive system was designed and constructed with a controller. The drive consists of several distinct sub systems as illustrated in Fig. 8: 4-phase IGBT inverter (two switch per phase type), 8/6 SR motor (4kW, 415V, 9A, $1500 \mathrm{rpm}$, four phase), the DSP board, A/D converters, and the interfaces for signal input and output (currents, voltages, position and gate signals for IGBT's).

The controller consists of a high-speed DSP chip (ADSP-21 020) with on board memory. The DSP performs the fuzzy logic based rotor position estimation in addition for providing full control of the motor via the power inverter. Inputs to the digital signal processor include the current and the voltage of each phase. To sample four phase voltages and currents simultaneously, eight A/D converters were used, and in order to provide greater digital conversion accuracy, a programmable gain amplifier was utilized to adjust the gain
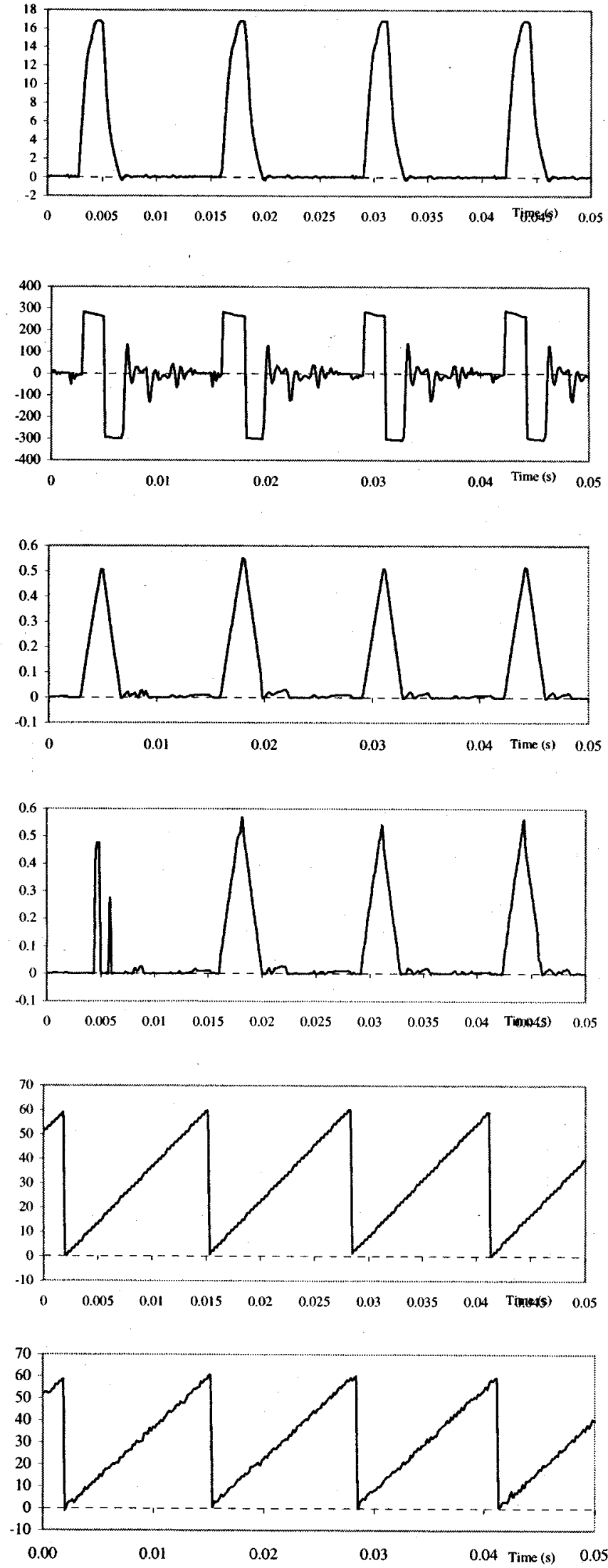

Fig. 9. Measured current, voltage, estimated flux linkage, predicted flux linkage of phase A, measured and estimated position, respectively, from the top, $810 \mathrm{rpm}$.

of the voltage signal. A shaft encoder was used to provide a reference for checking the estimated position. 


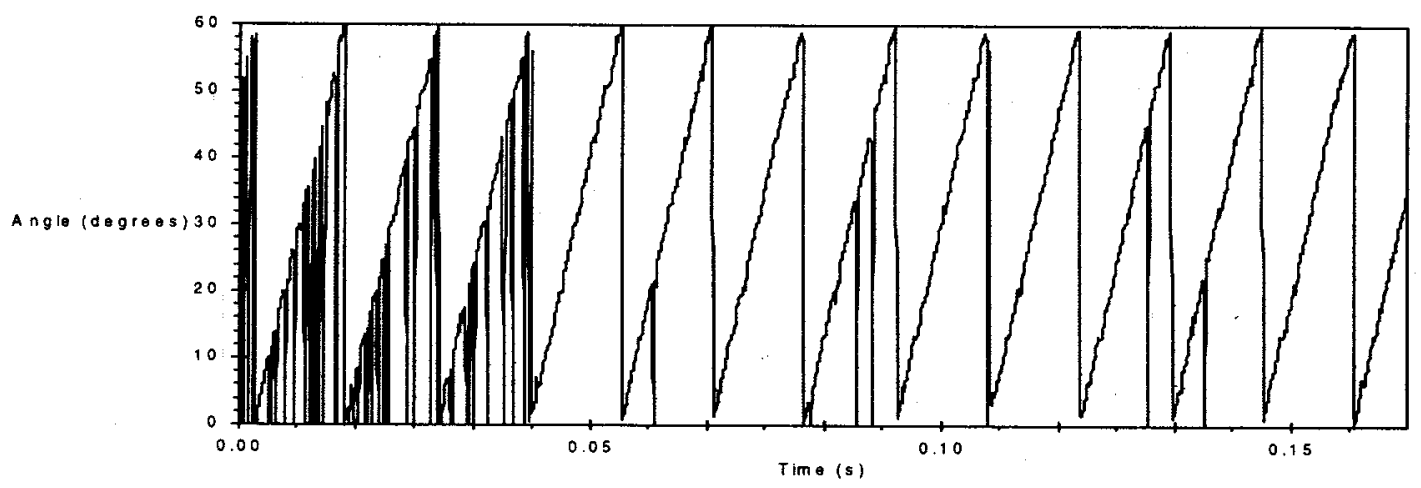

(a)

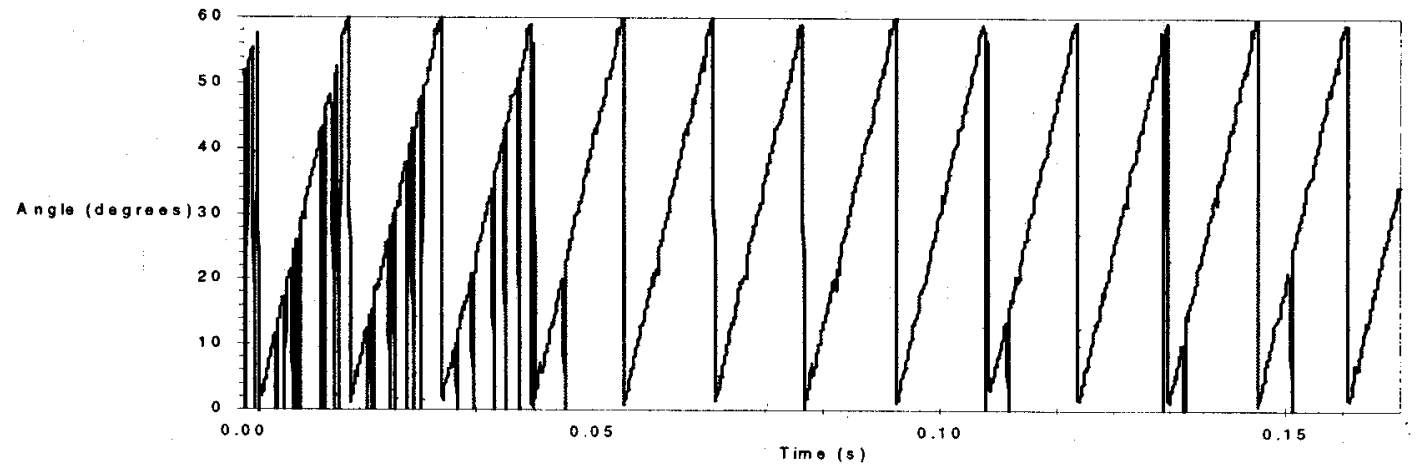

(b)

Fig. 10. Predicted and next step ahead predicted angles (in degrees).

The actual operating effects of the SR motor drive include: mutual inductance between motor phases, parameter variation of motor inductances and resistances, asymmetrical inductance variation in the motor phases, variation in the magnetization curves in each of the phases, and effects on the motor waveforms of eddy currents (which can distort the phase current, especially during current transients).

Therefore, experimental waveforms, which will be affected by all the above effects, should be used to verify the ability of the sensorless scheme to operate with a real SR motor. Using the experimental hardware, a wide range of operating modes and conditions were applied to the test motor. A few distinct operating results are presented in this paper: single pulse operation at steady-state speed, acceleration from zero speed, and zero/slow speed operation.

1) Single Pulse Mode Operation: In Fig. 9, the test results are given for the single pulse mode of the motor with a steadystate speed of $810 \mathrm{rpm}$. At high speeds, the back emf during pole overlap will become higher than the dc supply voltage. Therefore, the current does not reach the chopping mode current level (but is greater than the rated current) during the phase conduction period. Note that justification of "high" or "low" speed operation depends upon the machine details. For the motor used in this paper, low, base and high speeds correspond to the chopping mode, the changeover speed of $650 \mathrm{rpm}$ and the single pulse mode, respectively.

It can also be seen in the figure that, in the single pulse mode, the current waveform is regular and the flux linkage has a simple profile, a quasitriangular wave-shape. Therefore, it was found that the fuzzy predictors of flux linkage have a short learning period with these flux linkage waveforms. This learning period was less than 200 iterations, corresponding to $46 \%$ of one revolution.

The reference position measured by the encoder had an 8-bit output, which means that 256 discrete positions per revolution can be measured, which corresponds to $256 / 6 \cong 43$ discrete points per electrical cycle. Therefore, in calculating the position error in the test results, the encoder measurement will normally have some quantization error, and thus not provide a very accurate reference. Nevertheless, it was taken to be the true rotor angle when comparisons were made between the estimated and the measured angle.

The predicted and next step ahead predicted angles for the test in Fig. 9 is given in Fig. 10. It was found that approximately 240 iterations were needed for the initial learning period. This was equivalent to $52 \%$ of one revolution of the rotor. In comparison with the flux linkage prediction of this test, it is seen that the learning period is slightly longer, due to the higher estimation error of position in comparison to the estimation error of flux linkage in this test.

2) Chopping Mode Transient Operation: To verify the operation of the method during transient operation, another set of experimental results is given in Fig. 11 during a transient start-up from zero speed to $346 \mathrm{rpm}$. As can be seen in the figure, the phase A motor current increases during the starting period and exceeds the rated current of 9A. However, when the motor approaches to the steady state speed of $346 \mathrm{rpm}$, the currents become significantly lower due to the decreased acceleration torque. This operation presents a more difficult problem for the position estimation due to following two main reasons. 

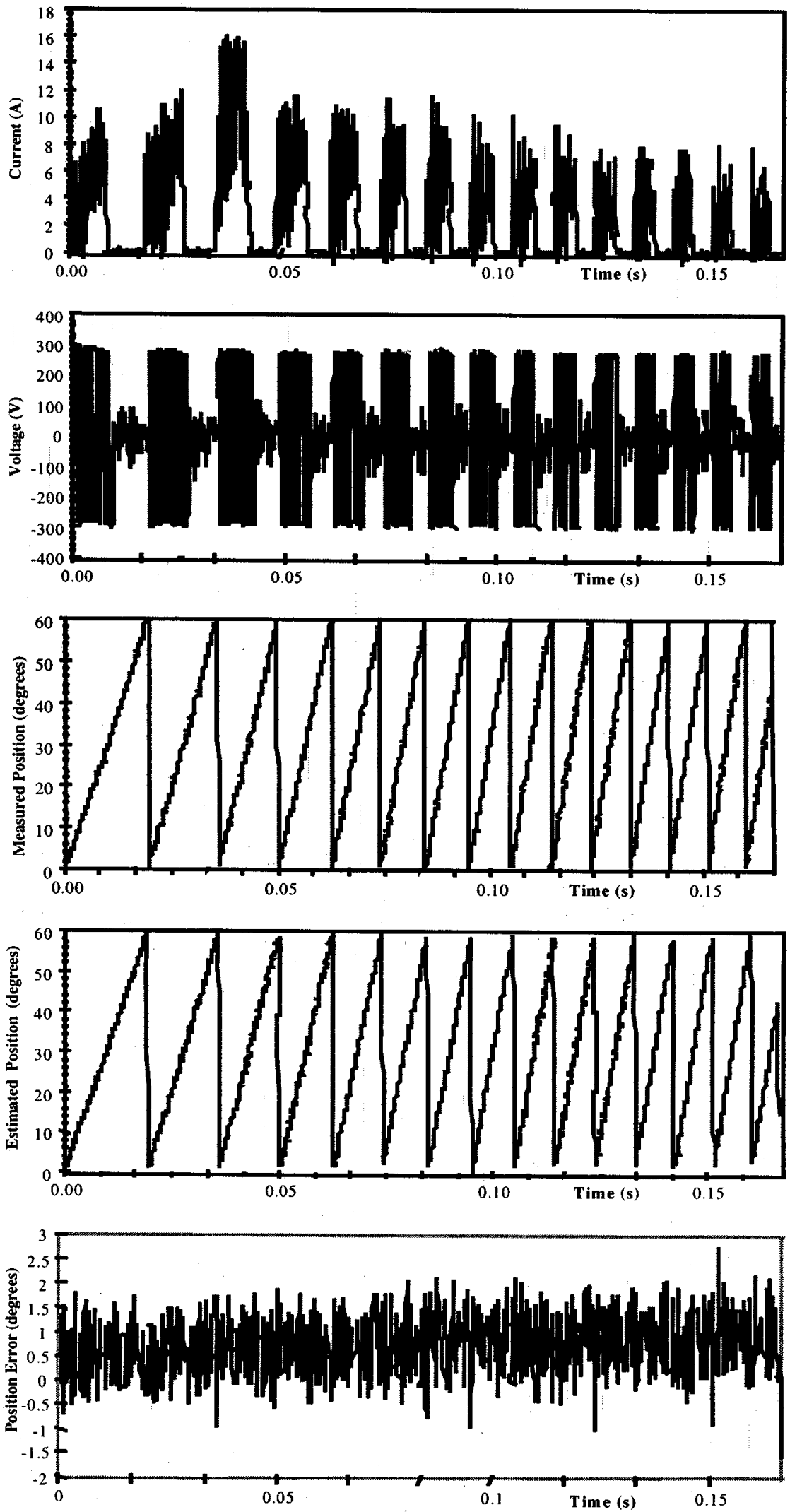

Fig. 11. Results of the transient start up with hysteresis current control: current, voltage, measured position, estimated position, and position error.

1) Not all the components of the waveforms are captured by the A/D converter at the specified constant sampling frequency of $6 \mathrm{kHz}$.
2) The predictors of flux linkage and rotor position, which are used to lessen errors in the flux linkage and position estimation, are not as effective during transient modes of 


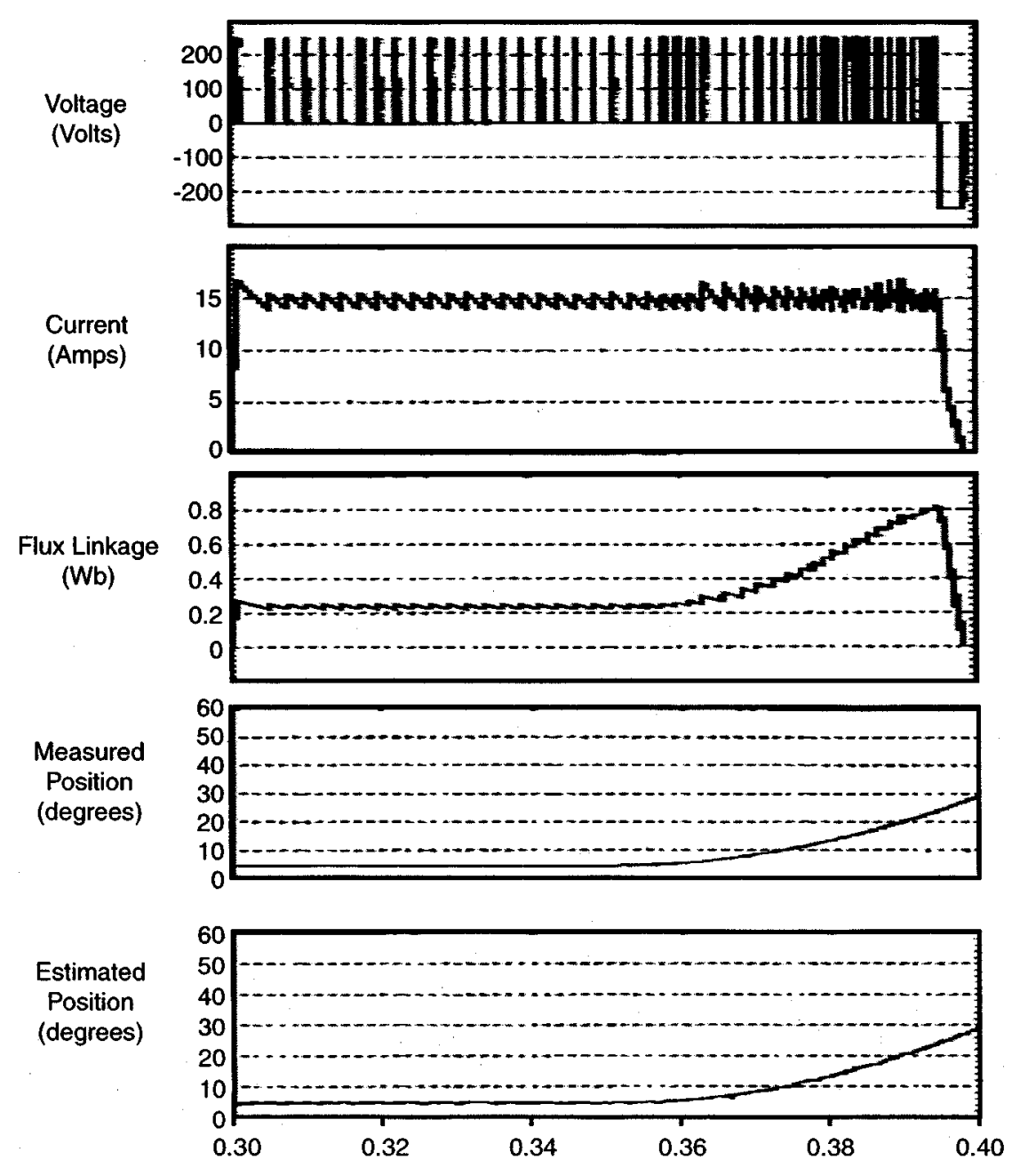

Fig. 12. Zero/low speed test.

operation as stated in Section III-A. This is because of the learning period always required when the motor waveforms vary. Therefore, the confidence in the predicted values will not be as high in the transient mode of operation.

In the transient mode of operation, the average angle error is somewhat higher. The performance has suffered due to the higher flux linkage estimation errors, and the trajectory of rotor position continuously changes in contrast to the linear variation of the previous steady-state speed tests. However, the estimated angle follows the measured position closely throughout the test.

3) Zero/Low Speed Operation: In this test the motor is initially stalled with a high inertial mechanical load that is thereafter slowly accelerated. The conditions of this test allow the sensorless position estimation algorithm to be tested for a number of important conditions. This includes zero speed operation (seen from $t=0.3 s$ to $t=0.35 s$ ), slow acceleration, and low speed (seen from $t=0.35 s$ onwards).

The plot of the phase current, phase voltage, phase flux linkage, as well as measured and estimated rotor position, are shown together for one motor phase in Fig. 12. At $t=0.3 \mathrm{~s}$ the rotor is stalled, and it can thus be seen that the rotor angle remains constant. However at $t=0.35 \mathrm{~s}$ the rotor is again accelerated with a low acceleration rate, and the rotor position again gradually increases.

It can be seen in the phase current waveforms of Fig. 12 that there are oscillations in the current hysteresis level even during zero and low speed. This is due to the bandwidth limitation of the hysteresis current controller. Furthermore it can be seen that the flux linkage changes when the rotor begins to accelerate from zero speed due to the change in incremental inductance with position.

The estimated angle that is seen in these results can be seen to always have some error, even though the rotor position is constant. This can be explained by the fact that the current is not constant but has variations due to the hysteresis control. This high frequency variation will not be completely captured by the A/D converters, leading to measurement errors. Furthermore noise and modeling errors that are always present will lead to the error seen in the rotor position.

A large amount of other experimental tests were performed on the motor to vigorously prove the performance and reliability of the fuzzy logic sensorless rotor position detection scheme under all conditions. Although two sets of the tests (steady state and transient) were presented above, Table II is provided to show the comparison of other distinctive test results, average of the absolute value of the position errors, maximum errors and cor- 
TABLE II

SUMMARY OF TEST RESULTS ON THE ERROR ANALYSIS

\begin{tabular}{|c|c|c|c|}
\hline OPERATION MODE & $\begin{array}{l}\text { Average of the } \\
\text { absolute value of } \\
\text { error (degrees) }\end{array}$ & $\begin{array}{l}\text { Max. Error } \\
\text { (degrees } \\
\text { electrical) }\end{array}$ & $\begin{array}{c}\text { Learning } \\
\text { Period } \\
\text { (no of iteration) }\end{array}$ \\
\hline \multicolumn{4}{|l|}{ STEADY-STATE OPERATION } \\
\hline $\begin{array}{l}\text { Low speed chopping mode test (command } \\
\text { current level of } 8 \mathrm{~A}, \pm 4 \mathrm{~A} \text { hysteresis), } 162 \\
\text { rpm, sampling frequency of } 6000 \mathrm{~Hz} \text {. }\end{array}$ & 0.48 & 1.55 & $\sim 400$ \\
\hline $\begin{array}{l}\text { Mid-speed chopping mode test (command } \\
\text { current level of } 10 \mathrm{~A}, \pm 2 \mathrm{~A} \text { hysteresis), } 326 \\
\text { rpm, sampling frequency of } 6000 \mathrm{~Hz} \text {. }\end{array}$ & 0.36 & 2.64 & $<400$ \\
\hline $\begin{array}{l}\text { High-speed chopping mode test (maximum } \\
\text { current level of } 10 \mathrm{~A}, \pm 4 \mathrm{~A} \text { hysteresis), } 606 \\
\text { rpm, sampling frequency of } 6000 \mathrm{~Hz} \text {. }\end{array}$ & 0.79 & 2.80 & $\sim 420$ \\
\hline $\begin{array}{l}\text { High-speed single pulse mode test (peak } \\
\text { current level of } 17 \mathrm{~A} \text { ), } 810 \mathrm{rpm} \text {, sampling } \\
\text { frequency of } 6000 \mathrm{~Hz} \text {. }\end{array}$ & 0.52 & 1.92 & $200-250$ \\
\hline \multicolumn{4}{|l|}{ TRANSIENT OPERATION } \\
\hline Start-up from standstill to $346 \mathrm{rpm}$ & 0.82 & 2.87 & \\
\hline $\begin{array}{l}\text { Change in load while running in chopping } \\
\text { mode at } 454 \mathrm{rpm}\end{array}$ & 1.11 & 2.93 & 280 \\
\hline
\end{tabular}

responding learning periods in each test [22], [23]. When the quantization error in the measured position is taken into account, the position error presented in the previous graphs and in Table II are not high. The operation of the motor has not been effected due to the small error in the position estimation. However, it should be emphasize here that the position estimation error can be reduced further if the sampling frequency and/or number of fuzzy-regions are increased. In addition to this, it should be remembered that measured reference position may also be in error.

\section{A. Effectiveness of Fuzzy Predictors in Practical Drive Operation}

The practical SR motor drive often has the problem of high amplitude impulsive type noise caused by switching or commutation of high amplitude currents in the inverter circuit. The commutated current waveforms have short rise and fall times, and thus contain significant amounts of energy at high frequencies. This radiated energy can be transmitted through parasitic stray capacitances to the control, interface, and measurement circuitry.

The characteristic feature of this generated noise is that it can have high amplitude during the switching of a power device. However, this noise is only seen during the switching instant. Therefore, the coupled noise in the control and current and voltage measurement circuits may have high amplitude, but be transient in nature. This type of high amplitude impulsive type noise is difficult to suppress efficiently [26]. However, the fuzzy predictive filters of flux linkage and angle as described above were developed to successfully lower the effect of impulsive noise for the practical operation of the sensorless position estimation scheme.

In Fig. 13, a demonstration of the fuzzy predictive filter's effectiveness is shown. Firstly, these figures show estimated flux linkage and angle derived from experimentally measured waveforms of current and voltage with the motor drive running at $670 \mathrm{rpm}$. It can be seen in the figures, that high amplitude error pulses occur in both the estimated flux linkage waveform and the estimated rotor position waveform.

In Fig. 13(a), the waveform of the flux linkage estimated from the measured current and voltages are shown, with a triangle representing each point where the flux is estimated from the current and voltage measurements. In the figure, a flux linkage waveform with high noise error can also be seen. The estimated flux linkage waveform with error is input to the flux linkage predictor instead of the actual estimated flux. It can be seen that the points with high-level noise have effectively been replaced by predicted values. It should be noted that if the predictor could not remove the erroneous value, then due to the operation of integration, all future values of the estimated flux linkage would carry this error.

Fig. 13(b), the waveforms are shown of the measured encoder angle and the estimated angle with impulsive type noise (at different test times, but with the same conditions as the flux linkage test). In the results, a triangular point shows the measured encoder positions at each sample time. In this test, the estimated angle that has been corrupted with high amplitude noise pulses is input to the angle predictor. It can be seen that the noise in the estimated angle is effectively removed in the filtered angle value. The filtered value can thus be used instead of the estimated value to reduce the effect of switching noise.

The results above have shown that when the fuzzy logic based predictive filters of flux linkage and angle are used, the high error pulses from sources such as switching noise are effectively eliminated, which leads to a more robust and stable motor drive operation.

\section{CONCLUSION}

A novel fuzzy logic based rotor position detection technique is explained and implementation details are given, which provides an alternative way of measuring the rotor position in SR motor drives. The experimental tests verified that the new scheme can successfully and vigorously predict the rotor angle of the practical SR motor under the real operating conditions. 


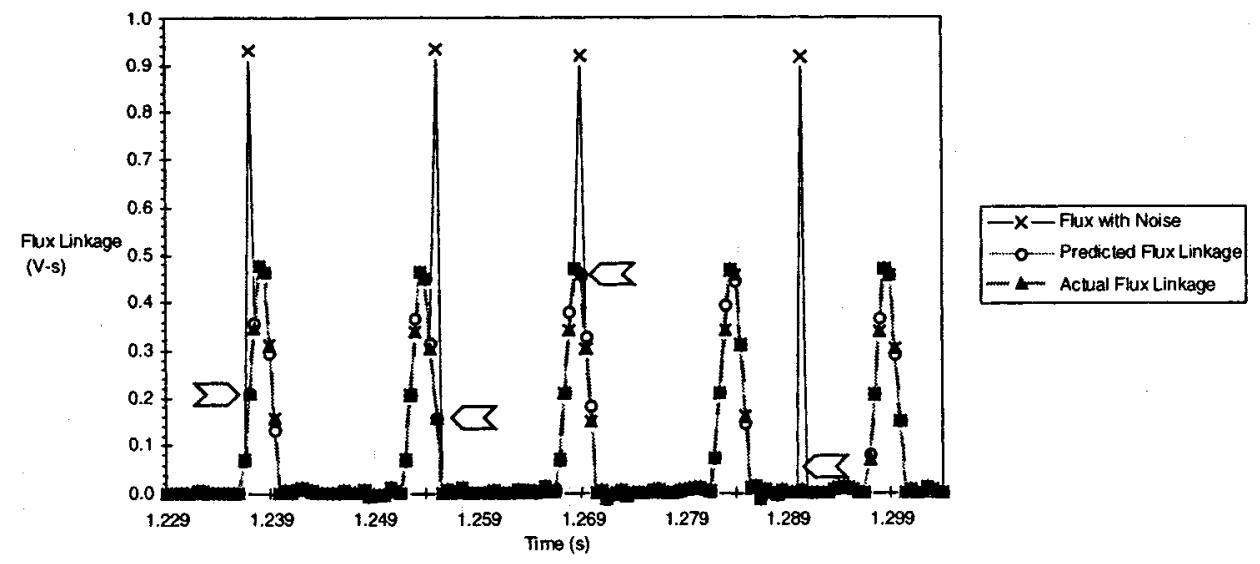

(a)

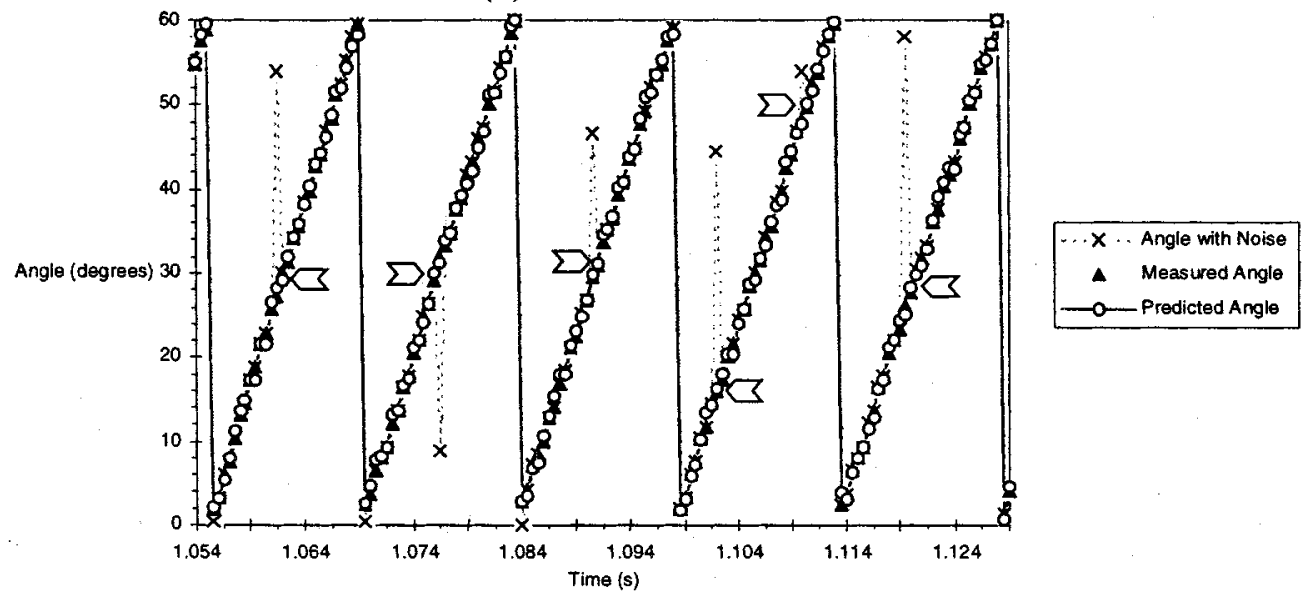

(b)

Fig. 13. Error elimination ability of fuzzy logic-based predictors: (a) flux linkage and (b) rotor position. The points with arrows highlight the iterations where the predicted value can be used instead of the estimated angle to lessen the effect of switching noise.

TABLE III

Principal Improvements of the New Scheme Over the Most Sophisticated Methods to Date: The Model Based Schemes

\begin{tabular}{l|c|c}
\hline Parameter & New Fuzzy Logic Based Scheme & Model Based Schemes \\
\hline Speeds & All including stall and start-up & $\begin{array}{c}\text { Not demonstrated for stall and } \\
\text { start-up }\end{array}$ \\
\hline $\begin{array}{l}\text { High Robustness and } \\
\text { Reliability }\end{array}$ & Demonstrated & $\begin{array}{c}\text { Not demonstrated } \\
\text { Mathematical model } \\
\begin{array}{l}\text { Includes non-linear } \\
\text { saturation }\end{array}\end{array} \quad$ None required \\
$\begin{array}{l}\text { Includes all real time } \\
\text { operating effects }\end{array}$ & Yes & Accurate state space model \\
\hline $\begin{array}{l}\text { Requires test signals } \\
\text { Can be used under all } \\
\text { current control } \\
\text { conditions }\end{array}$ & Yes & No \\
\hline
\end{tabular}

Since there is no starting difficulty in the motor drive and no difficulty at zero speed operation, the motor drive can be operated in four-quadrants. It was shown that in general, the fuzzy logic based prediction algorithm had a fast learning period of approximately 200-250 iterations for simpler waveforms, such as the rotor angle trajectory and the flux linkage in the single pulse mode. The predictors of flux linkage and angle were also shown to be effective. The learning period became longer for highly nonlinear waveforms, such as the flux linkage in chopping mode.

The position estimator implemented in this research does not have restrictions seen in other schemes, as it can be used under 
all operating speeds and conditions, including transients, standstill, and start-up. It also does not require any external testing circuitry or test signals.

The tests shown were taken with the A/D converter sampling frequency limited to $6000 \mathrm{~Hz}$. This demonstrates the ability of the scheme to work with a relatively long iteration time of 166.7 $\mu \mathrm{s}$. The average processing time of the position estimation routine is approximately $33 \mu$ s (the cycle time varies slightly according to which particular subroutines are used in the fuzzy prediction and fuzzy rule processing routines). The processing time can be further reduced by optimization of code and full assembly programming. The advantages made available by this scheme are summarized in Table III.

\section{REFERENCES}

[1] A. Cheok and N. Ertugrul, "Sensorless rotor position detection techniques in switched reluctance motor drives," in Proc. Australasian Universities Power Eng. Conf., Perth, Australia, Sept. 1995, pp. 27-29.

[2] P. P. Acarnley, R. J. Hill, and C. W. Hooper, "Detection of rotor position in stepping and switched reluctance motors by monitoring of current waveforms," IEEE Trans. Ind. Electron., vol. IE-32, pp. 215-222, Feb. 1985.

[3] M. Ehsani, I. Husain, and A. B. Kulkarni, "Elimination of discrete position sensor and current sensor in switched reluctance motor drives," IEEE Trans. Ind. Applicat., vol. IA-28, pp. 128-135, Jan./Feb. 1992.

[4] S. K. Panda and G. A. J. Amaratunga, "Waveform detection technique for indirect rotor-position sensing of switched-reluctance motor drives-Part 1: Analysis," Proc. Inst. Elect. Eng. B., vol. 141, no. 1, pp. 80-88, 1993.

[5] J. P. Lyons, S. R. MacMinn, and M. A. Preston, "Flux/current methods for SR MOTOR rotor position information," in Proc. Conf. Rec. IEEE Ind. Applicat. Soc. Annu. Meeting, 1991, pp. 482-487.

[6] W. F. Ray and I. H. Al-Bahadly, "A sensorless method for determining rotor position for switched reluctance motors," in Proc. IEE 5th Int. Conf. Power Electron. Variable Speed Drives, 1994, pp. 13-17.

[7] M. Ehsani and I. Husain, "Rotor position sensing in switched reluctance drives by measuring mutually induced voltages," in Proc. Conf. Rec. IEEE Ind. Applicat. Soc. Annu. Meeting, 1992, pp. 422-429.

[8] Gallegos-Lopez, P. C. Kjaer, and T. J. E. Miller, "High-grade position estimation for SRM drives using flux linkage/current correction model," IEEE Trans. Ind. Applicat., vol. 35, pp. 859-869, Jul./Aug. 1999.

[9] A. Cheok and N. Ertugrul, "High robustness and reliability of a fuzzy logic based angle estimation algorithm for practical switched reluctance motor drives," in Proc. IEEE Power Electron. Spec. Conf., Fukuoka, Japan, May 1998

[10] C. Elmas and H. Zelaya-Da La Parra, "Position sensorless operation of a switched reluctance drive based on observer," in Proc. IEEE EPE Conf., 1993, pp. 82-87.

[11] Lumbsdaine and J. H. Lang, "State observers for variable reluctance motors," IEEE Trans. Ind. Electron., vol. 37, no. 22, pp. 133-142, 1990.

[12] Y. J. Zhan, C. C. Chan, and K.T. Chau, "A novel position and velocity observer for robust control of switched reluctance reluctance motors," IEEE Power Electron. Spec. Conf., pp. 1315-1321, 1998.

[13] P. Vas, Artificial-Intelligence-Based Electrical Machines and Drives: Application of Fuzzy, Neural, Fuzzy-Neural, and Genetic-Algorithm-Based Techniques. New York: Oxford, 1999.

[14] A. Cheok and N. Ertugrul, "A model free fuzzy logic based rotor position sensorless switched reluctance motor drive," in IEEE IAS Апnи. Meeting, 1996, pp. 76-83.

[15] L. X. Wang, Adaptive Fuzzy Systems and Control: Design and Stability Analysis. Englewood Cliffs, NJ: Prentice-Hall, 1994.

[16] G. C. D. Sousa and B. Bose, "A fuzzy set theory based control of a phasecontrolled converter DC machine drive," IEEE Trans. Ind. Applicat., vol. 30, pp. 34-44, Feb. 1994
[17] J. L. Castro, "Fuzzy logic controllers are universal approximators," IEEE Trans. Syst. Man Cybern., vol. 25, pp. 629-636, Apr. 1995.

[18] C. Elmas, S. Sagirogiu, I. Colak, and G. Bai, "Modeling of a nonlinear switched reluctance motor drive based on artificial neural networks," in Proc. IEE PEVSD, 1994, pp. 7-12.

[19] T. Frenz and D. Schroder, "Learning unknown nonlinearities using a discrete observer in combination with neural networks," in Proc. IEEE Power Electron. Spec. Conf., 1995, pp. 1800-1806.

[20] S. Abe and M. Lan, "Fuzzy rules extraction directly from numerical data for function approximation," IEEE Trans. Syst. Man Cybern., vol. 25, pp. 119-129, Jan. 1995.

[21] Lea and V. Kreinovich, "Intelligent control makes sense even without expert knowledge: An explanation," in Proc. APIC'95 (Suppl. Int. J. Reliable Comput.), El Paso, NM, 1995.

[22] A. Cheok and N. Ertugrul, "High robustness and reliability of a fuzzy logic based angle estimation algorithm for practical switched reluctance motor drives," IEEE Trans. Power Electron., to be published.

[23] A. Cheok, "A new fuzzy logic based rotor position estimation algorithm for switched reluctance motor drives," Ph.D. dissertation, Univ. Adelaide, Adelaide, Australia, Jan. 1998.

[24] G. C. Mouzouris and J. M. Mendel, "Dynamic nonsingleton fuzzy logic systems for nonlinear modeling," IEEE Trans. Fuzzy Syst., vol. 5, pp. 199-208, May 1997.

[25] J. P. Lyons, S. R. MacMinn, and M. A. Preston, "Flux/current methods for SRM rotor position estimation," in Proc. IEEE Ind. Applicat. Soc. Аnnu. Meeting, 1991, pp. 482-487.

[26] Papoulis, Probability, Random Variables, and Stochiastic Processes. New York: McGraw-Hill, 1984.

[27] S. Väliviita and O. Vainio, "Delayless differentiation algorithm and its efficient implementation for motion control applications," in Proc. IEEE Instrum. Meas. Technol. Conf., St Paul, MN, 1998, pp. 881-886.

[28] G. Gallégos-Lopez, P. C. Kjaer, and T. Miller, "A new sensorless method for switched reluctance motor drives," IEEE Trans. Ind. Applicat., vol. 34, pp. 832-840, July/Aug. 1998.

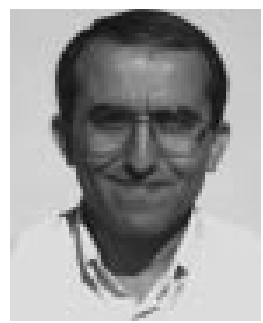

Nesimi Ertugrul (M'95) received the B.Sc. (Eng). and M.Sc. degress in electrical and in electronic and communication engineering from the Istanbul Technical University, in 1985 and 1989 respectively, and received Ph.D. degree from the University of Newcastle upon Tyne, United Kingdom, in 1993.

He joined the University of Adelaide, Australia in 1994. His research topics include rotor position sensorless operation of brushless permanent magnet and switched reluctance motors, real-time control of electrical machine drives and power electronics systems, alternative energy sources, electrical vehicles, and interactive computer-based teaching/learning systems involving object-oriented programming and data acquisition. He is on the Editorial Advisory Board of the International Journal of Engineering Education.

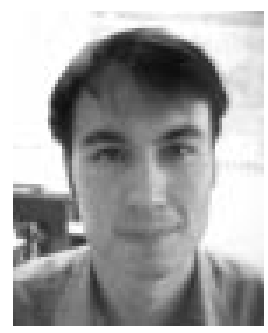

Adrian D. Cheok (M'93) received the B.Eng and $\mathrm{Ph} . \mathrm{D}$. degrees from the University of Adelaide, Adelaide, Australia, in 1993 and 1998 respectively.

From 1996 to 1998 , he was with Transmission and Distribution, Transportation Systems Center, Mitsubishi Electric Corporation, Amagasaki, Japan. Since 1998, he has been an Assistant Professor in the Department of Electrical Engineering, National University of Singapore, Singapore. His research interests include power electronics and motor drives, fuzzy logic and soft computing, nonlinear modeling and control, noise and EMI, embedded systems, and digital signal processing. 